\title{
Transcranial Magnetic Stimulation: An Automated Procedure to Obtain Coil-specific Models for Field Calculations
}

\author{
Madsen, Kristoffer Hougaard; Ewald, Lars; Siebner, Hartwig R.; Thielscher, Axel
}

Published in:

Brain Stimulation

Link to article, DOI:

10.1016/j.brs.2015.07.035

Publication date:

2015

Document Version

Peer reviewed version

Link back to DTU Orbit

Citation (APA):

Madsen, K. H., Ewald, L., Siebner, H. R., \& Thielscher, A. (2015). Transcranial Magnetic Stimulation: An

Automated Procedure to Obtain Coil-specific Models for Field Calculations. Brain Stimulation, 8(6), 1205-1208. https://doi.org/10.1016/j.brs.2015.07.035

\section{General rights}

Copyright and moral rights for the publications made accessible in the public portal are retained by the authors and/or other copyright owners and it is a condition of accessing publications that users recognise and abide by the legal requirements associated with these rights.

- Users may download and print one copy of any publication from the public portal for the purpose of private study or research.

- You may not further distribute the material or use it for any profit-making activity or commercial gain

- You may freely distribute the URL identifying the publication in the public portal 


\section{Transcranial magnetic stimulation:}

\section{An automated procedure to obtain coil-specific models for field calculations}

Kristoffer H. Madsen ${ }^{1,2}$, Lars Ewald ${ }^{1}$, Hartwig R. Siebner ${ }^{1,3}$, Axel Thielscher ${ }^{1,4,5}$

${ }^{1}$ Danish Research Centre for Magnetic Resonance, Centre for Functional and Diagnostic Imaging and Research, Copenhagen University Hospital Hvidovre, Denmark

${ }^{2}$ Cognitive Systems, DTU Compute, Technical University of Denmark, Kgs. Lyngby, Denmark

${ }^{3}$ Department of Neurology, Copenhagen University Hospital Bispebjerg, Denmark

${ }^{4}$ Biomedical Engineering Section, Technical University of Denmark, Kgs. Lyngby, Denmark

${ }^{5}$ Max-Planck Institute for Biological Cybernetics, Tübingen, Germany

Correspondence by: Kristoffer H. Madsen

Email: kristofferm@drcmr.dk

Phone: (+45) 38623407 


\begin{abstract}
Background: Field calculations for transcranial magnetic stimulation (TMS) are increasingly implemented online in neuronavigation systems and in more realistic offline approaches based on finite-element methods. They are often based on simplified and/or non-validated models of the magnetic vector potential of the TMS coils.
\end{abstract}

Objective: To develop an approach to reconstruct the magnetic vector potential based on automated measurements.

Methods: We implemented a setup that simultaneously measures the three components of the magnetic field with high spatial resolution. This is complemented by a novel approach to determine the magnetic vector potential via volume integration of the measured field.

Results: The integration approach reproduces the vector potential with very good accuracy. The vector potential distribution of a standard figure-of-eight shaped coil determined with our setup corresponds well with that calculated using a model reconstructed from x-ray images.

Conclusion: The setup can supply validated models for existing and newly appearing TMS coils.

\title{
Keywords
}

Transcranial magnetic stimulation, electric field calculations, magnetic vector potential, stimulation coils 


\section{Introduction}

The interest in electric field calculations for transcranial magnetic stimulation (TMS) has been steadily increasing in the last few years $[1,2]$. One motivation is to use the field calculations to better match the stimulation strengths across different subjects or also between different brain areas within the same subject [3-5]. In addition, more realistic field calculations based on the individual anatomy might also help to reveal the origins of the observed physiological stimulation effects [6]. The electric field is usually calculated assuming quasi-stationary conditions and solving the equation

$$
\boldsymbol{E}=-\nabla \varphi-\frac{\partial A}{\partial t}
$$

where $\boldsymbol{A}$ stands for the magnetic vector potential of the coil and $\varphi$ denotes an electric potential which depends on the conductivity distribution inside the head [6]. Characterizing the magnetic vector potential $\boldsymbol{A}$ is useful to capture the gross differences between coil types irrespective of the impact of the head geometry on current flow. Knowledge of $\boldsymbol{A}$ is also required for numerical approaches such as Finite-Element or Boundary-Element methods to solve equation 1 [6-9]. The vector potential is normally calculated based on models derived from the technical parameters of the coil such as the number and diameter of windings [10-13]. However, the accuracy of the resulting $\boldsymbol{A}$ field is usually not assessed, causing an error of unknown extent in the field calculations. Building accurate theoretical coil models is not straight-forward. Even when detailed information on the wire paths inside the coil housing is available, some effects such as the exact current distribution across the cross section of the relatively thick wires are difficult to capture. In addition, even small errors in the estimated distance of the wires to the outer surface of the coil housing will already result in marked changes of the absolute field strength at points that are close to the coil (including the brain surface). For this reason, we developed a novel approach that that determines the vector potential with high accuracy from automated measurements of the magnetic field $\mathbf{B}$ and can supply validated models for existing and newly appearing coils to the TMS community. 


\section{Material and methods}

First, a way to determine the magnetic vector potential from the magnetic field distribution of a coil via volume integration is derived. This is supplemented by a brief description of the implemented algorithm. Finally, the setup used for the automated field measurements is described.

\section{Obtaining the magnetic vector potential}

Biot-Savart's law provides the magnetic flux density $\boldsymbol{B}$ in the magneto-static case where the field is produced by a steady current distribution $\boldsymbol{J}$

$$
\boldsymbol{B}=\frac{\mu_{0}}{4 \pi} \int_{V} \frac{J(\tilde{\boldsymbol{r}}) \times(\boldsymbol{r}-\tilde{\boldsymbol{r}})}{|\boldsymbol{r}-\tilde{\boldsymbol{r}}|^{3}} \partial^{3} \tilde{\boldsymbol{r}}
$$

with $V$ denoting an integration over all of space, $\mu_{0}$ is the vacuum permeability, $\boldsymbol{r}$ and $\tilde{\boldsymbol{r}}$ are spatial position vectors, and $\times$ is the curl vector operator. Ampére's law and the divergence free property of the magnetic flux density can be stated as:

$$
\nabla \times \boldsymbol{B}=\mu_{0} \boldsymbol{J}, \quad \nabla \cdot \boldsymbol{B}=0,
$$

where $\nabla$ is the differential vector operator. From the definition of magnetic vector potential $\boldsymbol{A}$ and the freedom to choose a gauge, such as the Coulomb gauge, it equivalently holds that:

$$
\nabla \times \boldsymbol{A}=\boldsymbol{B}, \quad \nabla \cdot \boldsymbol{A}=0
$$

By analogy of $\boldsymbol{B}$ and $\mu_{0} \boldsymbol{J}$ equation 2 can therefore be rewritten to obtain the magnetic vector potential from an integration of the magnetic flux density over space:

$$
\boldsymbol{A}=\frac{1}{4 \pi} \int_{V} \frac{B(\tilde{\boldsymbol{r}}) \times(\boldsymbol{r}-\tilde{\boldsymbol{r}})}{|r-\tilde{r}|^{3}} \partial^{3} \tilde{\boldsymbol{r}}
$$

Rewriting this integral as two non-separable three dimensional convolutions of $\boldsymbol{B}$ for each component of $\boldsymbol{A}$ gives:

$$
\left[\begin{array}{l}
A_{x} \\
A_{y} \\
A_{z}
\end{array}\right](x, y, z)=\frac{1}{4 \pi} \iiint_{-\infty}^{\infty}\left(\begin{array}{c}
B_{y}(\tilde{x}, \tilde{y}, \tilde{z}) K_{z}(\tilde{x}, \tilde{y}, \tilde{z})-B_{z}(\tilde{x}, \tilde{y}, \tilde{z}) K_{y}(\tilde{x}, \tilde{y}, \tilde{z}) \\
B_{z}(\tilde{x}, \tilde{y}, \tilde{z}) K_{x}(\tilde{x}, \tilde{y}, \tilde{z})-B_{x}(\tilde{x}, \tilde{y}, \tilde{z}) K_{z}(\tilde{x}, \tilde{y}, \tilde{z}) \\
B_{x}(\tilde{x}, \tilde{y}, \tilde{z}) K_{y}(\tilde{x}, \tilde{y}, \tilde{z})-B_{y}(\tilde{x}, \tilde{y}, \tilde{z}) K_{x}(\tilde{x}, \tilde{y}, \tilde{z})
\end{array}\right) d \tilde{x} d \tilde{y} d \tilde{z},
$$


where the three kernels are defined as $\left[\begin{array}{l}K_{x}(\tilde{x}, \tilde{y}, \tilde{z}) \\ K_{y}(\tilde{x}, \tilde{y}, \tilde{z}) \\ K_{z}(\tilde{x}, \tilde{y}, \tilde{z})\end{array}\right]=\frac{1}{\sqrt{(x-\tilde{x})^{2}+(y-\tilde{y})^{2}+(z-\tilde{z})^{2}}}\left[\begin{array}{c}x-\tilde{x} \\ y-\tilde{y} \\ z-\tilde{z}\end{array}\right]$.

Assuming $\boldsymbol{B}$ is known in all of space (practically where it cannot be assumed to be zero) the integration can be discretized on a Cartesian grid (resolution of $2.5 \mathrm{~mm}$ in our case) and implemented efficiently using 3D fast Fourier transforms. The actual measurements of $\boldsymbol{B}$ can have arbitrary sampling resolutions and are regridded prior to numerical integration. As the integral covers the entire space, a combined cubic inter- and extrapolation scheme is used. This approach is feasible because the weighting of the integral drops off quickly with distance and the field is smooth. It is assumed that $\boldsymbol{B}=0$ at positions further away than twice the distance of the furthest measured point. Additionally, the measured planes are mirror reflected along a plane through the coil center and parallel to the main plane of the coil. The symmetry plane is easily defined for flat coils as considered here. Bended coils may require several symmetry planes and adaptations of the interpolation schemes. Please note that the integration step is rather robust to moderate misspecifications in the extrapolation procedure (see Fig. 1C) as the impact of remote magnetic fields level off quickly (eq. 5). The reconstructed $\boldsymbol{A}$ field in the measured volume of interest will thus be still accurate.

\section{Measurement setup}

The custom-made setup consists of a field probe, a plotter to position the probe in $2 \mathrm{D}$, a custommade amplifier coupled to a AD-converter (PicoLog1216, Pico Technology, UK), and a PC (Fig. 2A). The control of the measurement device is accomplished via software written in Python 2.7 (www.python.org). The soundcard of the PC is used to control the stepper motors of the plotter. An area of $60 \times 45 \mathrm{~cm}^{2}$ can be covered with a step size of $0.4 \mathrm{~mm}$. Initial testing of the plotter verified that it allows for a reproducible and accurate positioning of the probe in $2 \mathrm{D}$. The height of the field probe relative to the coil plane is set manually using a vernier caliper.

Three rectangular wire loops $(5 \times 5 \mathrm{~mm})$ made of very thin isolated copper wire are attached to the tip of the field probe. They are arranged perpendicular to each other to pick up the three components of the magnetic field. For calibration, the induced voltages were compared with the 
magnetic flux values determined with a commercial flux meter (FW Bell model 9500, equipped with a SAF99-1802 axial Hall probe) in a spatially homogeneous oscillating magnetic field.

\section{Results and Discussion}

First, the computational approach for inter- and extrapolation of the magnetic flux density $\boldsymbol{B}$ on a regular grid and for obtaining the magnetic vector potential $\boldsymbol{A}$ by volume integration was validated. A model of the Magstim 70mm figure-8 coil was used, based on X-ray images and a linear dipole expansion as described in [12]. The measurement procedure was simulated by calculating $\boldsymbol{B}$ on a grid with $7 \mathrm{~mm}$ in-plane spacing at several distances to the coil plane. The dimensions of the planes were chosen to be twice the size of the coil dimensions in order to ensure that the main part of the $\boldsymbol{B}$ field was captured. The $\boldsymbol{A}$ field obtained by our method from this $\boldsymbol{B}$ field distribution was then compared with the $\boldsymbol{A}$ field calculated directly from the dipole model (red lines in Fig. 1A). Similarly, the impact of different in-plane measurement spacings was tested for a specific set of measured distances (blue lines in Fig. 1A). Overall, accurate reconstructions are obtained for measurement distances $\leq 20 \mathrm{~mm}$, resulting in acceptable measurement times in practice. The reconstructed $\boldsymbol{A}$ field is shown exemplarily in Fig. 1D for a plane with $10 \mathrm{~mm}$ distance from the coil. The same in-plane spacing and distance between measured planes was used as in the subsequent real measurements. It can be seen from Fig. 1B that the residual variance at this distance is approximately $0.5 \%$. This value improves further for planes measured further away from the coil. Figure 1C explores the impact of shifts of the measurement grid and misspecifications of the coil mirror plane on the accuracy of the reconstruction. These results show that the procedure is relatively robust to these effects, indicating that a reasonable accuracy can be expected in practice.

The results of the real measurements are shown in Figure 2, with measurements scaled to the maximal output of $141 \cdot 10^{6} \mathrm{~A} / \mathrm{s}$ of the Magstim Rapid stimulator [11, 14, 15] for the used coil. The automated data acquisition for a single plane with $30 \times 18 \mathrm{~cm}^{2}(7 \mathrm{~mm}$ spacing, $2 \mathrm{~Hz}$ frequency) required 10 minutes. The positioning along the $3^{\text {rd }}$ dimension had to be done manually. While this increased the overall measurement time, it helped to limit the technical complexity. Still, 
actuating the $3^{\text {rd }}$ axis is a way to improve the setup in the future. The measured $\boldsymbol{B}$ field after interpolation is exemplarily shown in Figure $2 \mathrm{~B}$, again at $10 \mathrm{~mm}$ distance to the coil. Figure 2D depicts the reconstructed $\boldsymbol{A}$ field in the same plane. Please note that both the overall distribution and the peak value are very close to that determined with the dipole model used for Figure 1D. Specifically, the correlation coefficient between the modeled and the measured field taking into account the direction by evaluating over all three directions was 0.976 , and the optimal scaling factor to minimize the difference between the fields in a least-squares sense was 1.036. Figure $2 \mathrm{C}$ shows $\boldsymbol{A}$ in a plane perpendicular to the coil plane and aligned parallel to the main current direction in the center of the coil.

Both the simulation results and the $\boldsymbol{A}$ field determined for the real coil demonstrate that our new method reconstructs the magnetic vector potential very accurately. While the simulation results partly depend on our specific computational approach, they provide also a general rule-of-thumb on the sampling density required for field mapping of TMS coils. For coils of different size, we suggest to linearly scale the sampling density with the coil size relative to the one measured here. Our volume interpolation approach assumes mirror symmetry of the magnetic field with respect to the center plane of the coil. It was verified in simulations that it is robust to inaccuracies in choosing that plane. This also indicates that the field of coils with slight bends will likely be accurately reconstructed. However, we aim at further validating and (if necessary) extending the approach once we have measured bent coils.

To summarize, our approach will be useful to determine accurate $\boldsymbol{A}$ field distributions for the TMS community which can be used to validate the coil models embedded in, e.g. neuronavigation systems for field visualization [3] (the measurements will be linked on www.simnibs.org). In addition, they can be directly fed into field calculations based on numerical methods [16]. By that, it is ensured that both the coils and the head geometries are accurately represented in the simulations.

\section{Acknowledgements}

This work was supported by the Lundbeckfonden (Grant of Excellence "ContAct" R59-5399 to Hartwig Roman Siebner; Project grant R118-A11308 to Axel Thielscher) and by a Novo Nordisk Foundation Interdisciplinary Synergy Grant ("BASICS"). 


\section{References}

1. Rusconi, E. and S. Bestmann, On tickling brains to investigate minds. Cortex, 2009. 45(9): p. $1021-4$.

2. Sparing, R., M.D. Hesse, and G.R. Fink, Neuronavigation for transcranial magnetic stimulation (TMS): where we are and where we are going. Cortex, 2009. 46(1): p. 118-20.

3. Julkunen, P., et al., Within-subject effect of coil-to-cortex distance on cortical electric field threshold and motor evoked potentials in transcranial magnetic stimulation. Journal of Neuroscience Methods, 2012. 206(2): p. 158-164.

4. Roth, Y., et al., Motor cortex activation by H-coil and figure-8 coil at different depths. Combined motor threshold and electric field distribution study. Clinical Neurophysiology, 2014. 125(2): p. 336-343.

5. Trillenberg, P., et al., Variation of stimulation intensity in transcranial magnetic stimulation with depth. Journal of Neuroscience Methods, 2012. 211(2): p. 185-190.

6. Thielscher, A., A. Opitz, and M. Windhoff, Impact of the gyral geometry on the electric field induced by transcranial magnetic stimulation. Neuroimage, 2011. 54(1): p. 234-43.

7. Wagner, T.A., et al., Three-dimensional head model simulation of transcranial magnetic stimulation. IEEE transactions on bio-medical engineering, 2004. 51(9): p. 1586-1598.

8. De Lucia, M., et al., Diffusion tensor MRI-based estimation of the influence of brain tissue anisotropy on the effects of transcranial magnetic stimulation. NeuroImage, 2007. 36(4): p. 11591170.

9. Nummenmaa, A., et al., Comparison of spherical and realistically shaped boundary element head models for transcranial magnetic stimulation navigation. Clinical Neurophysiology, 2013. 124(10): p. 1995-2007.

10. Ravazzani, P., et al., Magnetic stimulation of the nervous system: induced electric field in unbounded, semi-infinite, spherical, and cylindrical media. Annals of Biomedical engineering, 1996. 24(5): p. 606-616.

11. Thielscher, A. and T. Kammer, Linking Physics with Physiology in TMS: A Sphere Field Model to Determine the Cortical Stimulation Site in TMS. NeuroImage, 2002. 17: p. 1117-1130.

12. Thielscher, A. and T. Kammer, Electric field properties of two commercial figure-8 coils in TMS: calculation of focality and efficiency. Clinical Neurophysiology, 2004. 115(7): p. 1697-1708. 
13. Deng, Z.D., S.H. Lisanby, and A.V. Peterchev, Electric field depth-focality tradeoff in transcranial magnetic stimulation: Simulation comparison of 50 coil designs. Brain Stimulation, 2013. 6(1): p. 1-13.

14. Ilmoniemi, R.J., J. Ruohonen, and J. Karhu, Transcranial magnetic stimulation - A new tool for functional imaging of the brain. Critical Reviews In Biomedical Engineering, 1999. 27(3-5): p. 241-284.

15. Kammer, T., et al., Motor thresholds in humans. A transcranial magnetic stimulation study comparing different pulseforms, current directions and stimulator types. Clinical Neurophysiology, 2001. 112: p. 250-258.

16. Windhoff, M., A. Opitz, and A. Thielscher, Field calculations in brain stimulation based on finite elements: An optimized processing pipeline for the generation and usage of accurate individual head models. Human Brain Mapping, 2013 34: p. 923-935. 

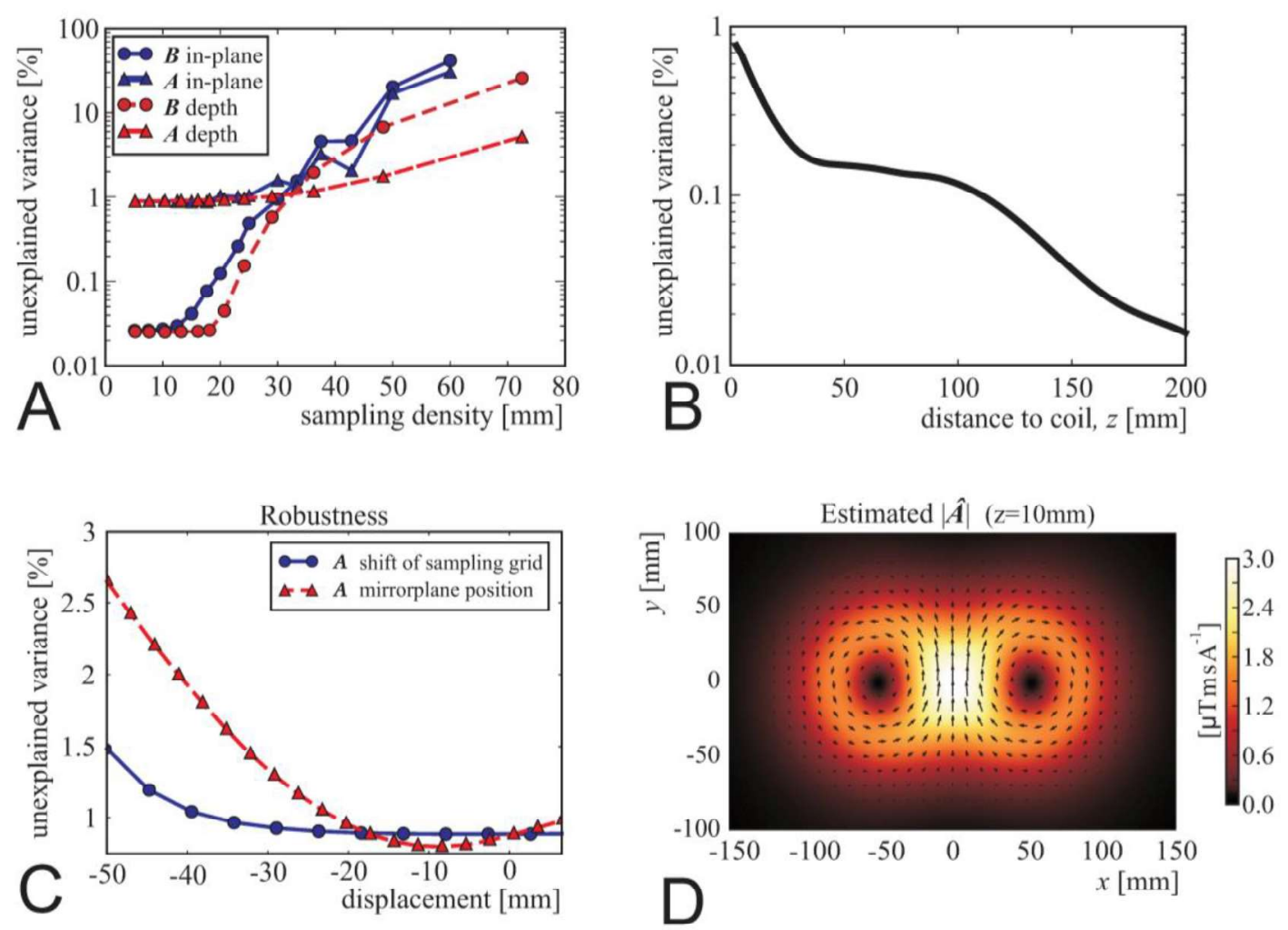

Figure 1: Validation of our approach based on a dipole coil model. A) Accuracy of the reconstruction of the $\boldsymbol{B}$ and $\boldsymbol{A}$ fields as function of in-plane $(x, y)$ resolution and depth resolution $(z)$. The estimated fields based on interpolation and volume integration are compared to field values obtained directly from the dipole model. The in-plane resolution is kept fixed at $7 \mathrm{~mm}$ (like in the real measurement) when obtaining the depth sampling curve (red lines). When varying the in-plane resolution, the distances between planes is kept constant (as used in the real measurements): $\mathrm{z}=3.5 \mathrm{~mm}, 4.9 \mathrm{~mm}, 7.7 \mathrm{~mm}, 12.6 \mathrm{~mm}, 17.8 \mathrm{~mm}, 23.5 \mathrm{~mm}, 32.8 \mathrm{~mm}, 44.5 \mathrm{~mm}, 65.2 \mathrm{~mm}$ and $81.3 \mathrm{~mm}$. B) Residual variance as function of the distance to the coil, with values normalized to the squared Frobenius norm of field summed over all positions at a distance of $10 \mathrm{~mm}$ to the coil $\left(\right.$ i. e. $\left.\frac{\|\boldsymbol{A}(z)-\widehat{A}(z)\|_{F}^{2}}{\|\boldsymbol{A}(z=10 \mathrm{~mm})\|_{F}^{2}}\right)$. This was favored over a perplane normalization to account for the fact that the amount of relative error is rendered increasingly unimportant the smaller the absolute field values get with increasing distance to the coil. The distance of $10 \mathrm{~mm}$ was chosen as a lower limit of the coil-cortex distance reached in practice. C) Curves indicating the robustness to misspecification of the sampling grid and mirror plane. The displacement of the sampling grid corresponds to misspecifying the center of the sampling grid in the $x y$-plane, therefore a negative value of $10 \mathrm{~mm}$ corresponds to having specified the center of the grid at $(x=-5 \sqrt{2} \mathrm{~mm}, y=-5 \sqrt{2} \mathrm{~mm})$ instead of $(x=0 \mathrm{~mm}, y=0 \mathrm{~mm})$, note that due to the symmetry of the coil positive and negative displacement expected to yield similar results. The other curve indicates the effect on accuracy of misspecification of the mirror reflection plane used in the extrapolation procedure. Here 0 would 
correspond to having specified the mirror plane in the center of the coil, note that the highest accuracy is actually obtained by specifying the mirror plane slightly further away $(\sim 10 \mathrm{~mm})$ than expected. This is caused by the interpolation scheme tending to underestimate the field inside the coil, which can be compensated to some degree by increasing the thickness of the coil. D) The reconstructed $\widehat{A}$ field at a distance of $10 \mathrm{~mm}$ to the coil with arrows indicating the in-plane direction of the vector field, note that the coil considered has no $z$-component of the $\boldsymbol{A}$ field.
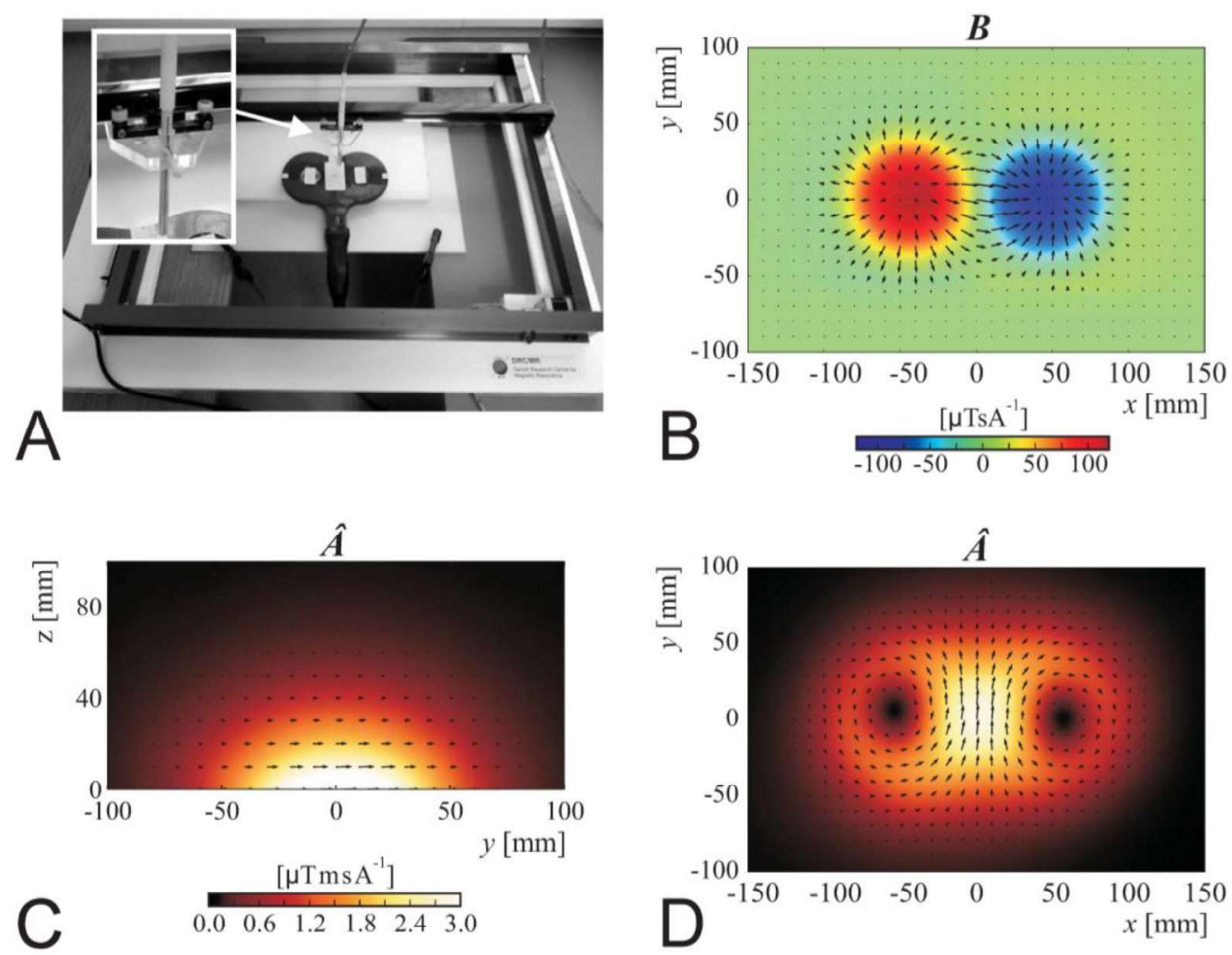

Figure 2: A) Measurement setup. The inset shows the three-dimensional measurement probe. B) Measured $\boldsymbol{B}$ field of the Magstim 70mm figure- 8 coil at a distance of $10 \mathrm{~mm}$ to the coil. The $z$-component of the field is coded as colors. The arrows depict the two remaining components. $\quad$ C) Reconstructed $\boldsymbol{A}$ field (magnitude) in a plane perpendicular to the coil plane and aligned with main direction of A underneath the coil center. D) Reconstructed $\boldsymbol{A}$ field shown for the same plane as in panel B. The magnitude is coded as colors. Please note the very good correspondence with the field shown in Fig. 1D based on the dipole coil model. It should be noted at that point that the deviation stems from inaccuracies both in the dipole coil model and the measurements and by that rather indicated an upper boundary of the measurement error of our approach. 